\title{
Application of Support Vector Machine in Fault Diagnosis of Elevator Key Structures
}

\author{
Yan Dou',2,a, Lanzhong Guo ${ }^{1,2, a}$, Yunbo Li ${ }^{3}$ and Yunfei Zhu ${ }^{3}$ \\ ${ }^{1}$ Mechanical Engineering, Changshu Institute of Technology, 215500, China \\ 2 Jiangsu Province Elevator Intelligent Safety Key Construction Laboratory, 215500, China \\ ${ }^{3}$ Suzhou Rhine Elevator Manufacture Co., Ltd, 215500, China
}

\begin{abstract}
Elevator is an important equipment in special equipment, its operation reliability is related to the life safety of elevator passengers; when the elevator fails, how to timely accurate identification of fault types and judgment of fault causes are the key to engineering application and theoretical research. This paper mainly introduces the application of support vector machine in fault diagnosis of elevator key structures.
\end{abstract}

\section{Significance of fault diagnosis}

The elevators used today are highly integrated, modular, and intelligent because of the high degree of control systems. Elevators often have an operational failure, which puts forward higher technical requirements for the maintenance personnel's work. The difficulty factor for maintenance personnel to solve problems in a short period of time increases; For the traditional repair after the failure not only increase the maintenance costs, affect travel, and even lead to other elevator operation load increase, and accelerate the loss of elevator parts and so on.

The introduction of support vector machine for elevator monitoring system to accurately judge the possible defects of the elevator and predict the possible failure is the research focus of foreign elevator manufacturing companies, and the research on the preventive maintenance strategy of the elevator is of great significance.

\section{The mission and process of fault diagnosis in elevator control system}

The task of fault diagnosis of elevator control system can be divided into four aspects from low-level to advanced:

\subsection{Elevator fault modelling}

According to the prior information and the input/output relationship, the mathematical model of the elevator system failure is established as the basis for the fault detection and judgment of the elevator.

\subsection{Elevator fault detection}

From measurable or immeasurable estimating variables, an alarm can be issued to determine whether the operating system is malfunctioning or not, and if unexpected changes occur.

\subsection{Elevator fault separation and assessment}

When the elevator control system fails, the location of the fault source is given, and the cause of the failure is distinguished from that of the control execution part, the sensing part or the controlled object, which is the biggest disturbance. The elevator fault estimation is to calculate the magnitude and time of the fault while ascertaining the cause of the failure.

\subsection{Elevator faults classification, evaluation and decision-making}

To judge the severity of elevator failure and its impact on the system and development trend, different measures are taken for different working conditions.

\footnotetext{
a Corresponding author: jixiedouyan@cslg.edu.cn, guolz@cslg.edu.cn
} 


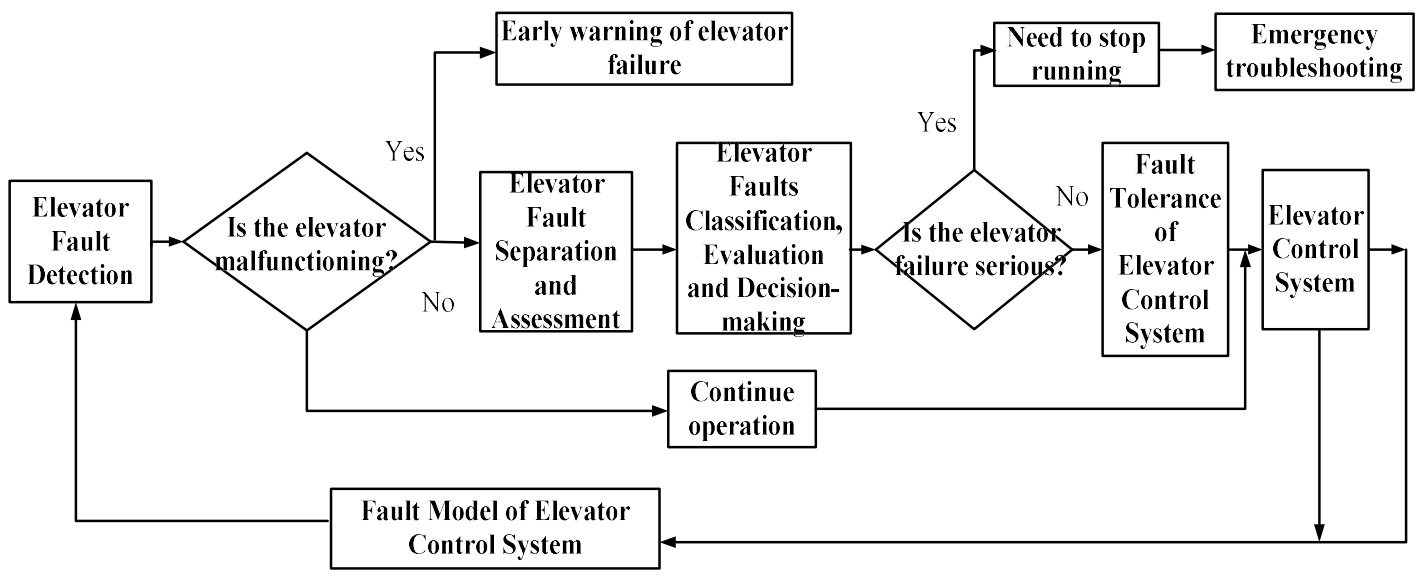

Figure 1. Fault Diagnosis Process of Elevator Control System.

\section{Support Vector Machine (SVM)}

Support Vector Machine (SVM) is a new machine algorithm developed on the basis of mathematical statistics theory, which adopts the principle of structural risk minimization and supervised learning. It can map a given low-dimensional sample data to a high-dimensional Hilbert space through non-linear transformation and perform linear analysis in this space. Its learning process is to solve quadratic programming problems with inequality constraints. Therefore, SVM has better generalization ability and generalization ability in dealing with non-linear data and small sample data.

Least Square Support Vector Machine (LSSVM) is a machine algorithm which is improved on the basis of Support Vector Machine (SVM) and applied to functional regression prediction and pattern recognition classification. It uses least squares system to construct loss function and uses equality constraints instead of inequality constraints of SVM. Finally, it transforms solving quadratic programming into solving linear equations.

\subsection{Least squares Support Vector Machine regression algorithms}

The advantage of LSSVM method is that it can solve large-scale problems, simplify the operation algorithm, improve the learning speed and reduce the calculation cost, at the cost of supporting the loss of sparsity and robustness of vector machine solutions.

Using Nonlinear Regression Function.

$$
f(x)=(w \bullet \varphi(x))+b
$$

To estimate the training sample set.

$$
D=\left\{\left(x_{k}, y_{k}\right) \mid k=1,2, \cdots, N\right\}
$$

$x_{k} \in R^{n}$ for input data, $y_{k} \in R$ for output data, $w$ for weight vector, $b$ for deviation.

The function estimation problem of LSSVM can be transformed into solving the following problems:

$$
\min _{w, b, e} J(w, e)=\frac{1}{2} w^{T} w+\frac{1}{2} \gamma \sum_{k=1}^{N} e_{k}^{2}
$$

\section{Fault diagnosis method of elevator closed loop control system based on Support Vector Machine}

In the closed-loop system of elevator, due to the existence of feedback, the input/output data are correlated, and the closed-loop control itself can restrain the fault to a certain extent.

How to determine the fast and effective fault diagnosis and isolation in the real-time closed-loop control of elevator control system is the focus and difficulty of the research.

\subsection{Fault diagnosis strategy of elevator closed loop control system based on Support Vector Machine}

When only one type of fault occurs in the closed-loop control system at a certain time, in order to realize the fault diagnosis of the closed-loop control system, firstly, a support vector machine identification model for fault diagnosis is established based on the measurement and known signals in the system, and then the fault occurrence and location are determined according to the diagnosis strategy.

Fault Diagnosis Strategy:

$\left\|e_{1}(k)\right\|=\left\|b(k)-b_{s \mathrm{~s} m}(k)\right\|=\left\{\begin{array}{l}\leq \delta_{1} \Rightarrow \text { fault }- \text { free elevator closed - loop control system } \\ >\delta_{1} \Rightarrow \text { failure of elevator closed loop control system }\end{array}\right.$

\subsection{Fault diagnosis of elevator closed loop control system based on Support Vector Machine}

The idea of fault diagnosis of elevator closed-loop control system based on support vector machine is to establish support vector machine identification model of the system and its large components through various measurements in the system. The output of each identification model is different from each measurement, and the fault is detected and located according to the different size. 
The idea of Support Vector Machine (SVM) method is to treat the system as a "single body". The internal mechanism of the system is unknown. The number of faults that can be diagnosed by SVM method depends on the number of actual measurements and the correlation between measurements.

\subsection{Elevator fault diagnosis strategy}

Fault Diagnosis Strategy of Elevator Closed-Loop Control System:

$$
\left\{\begin{array}{l}
\left\|e_{1}(k)\right\| \leq \delta_{1} \text { and }\left\|e_{1}(k)\right\| \leq \delta_{2} \Rightarrow \text { fault } \text { - free system } \\
\left\|e_{1}(k)\right\|>\delta_{1} \text { and }\left\|e_{1}(k)\right\| \leq \delta_{2} \Rightarrow \text { failure of feedback channel } \\
\left\|e_{1}(k)\right\|>\delta_{1} \text { and }\left\|e_{1}(k)\right\|>\delta_{2} \Rightarrow \text { forward channel failure }
\end{array}\right.
$$

\section{Design of elevator fault diagnosis system}

The main function of elevator fault diagnosis system is to complete elevator fault diagnosis using elevator operation data as data source. In this process, there are five problems to be solved:

How to get fault diagnosis information from elevator operation data; how to use knowledge base to diagnose; how to diagnose when knowledge base is incomplete; how to constantly supplement and update knowledge base; how to simplify the process of elevator fault diagnosis for maintenance personnel.

\subsection{Elevator fault diagnosis system}

Elevator fault diagnosis system should have the following modules: elevator task module, elevator fault acquisition module, elevator fault reasoning diagnosis module, elevator fault diagnosis results analysis module, elevator self-learning module.

\subsubsection{Elevator task module}

In order to ensure the availability of the system, the task module needs to diagnose the type of machine, the function of the system and components, and select the diagnostic method.

\subsubsection{Elevator fault acquisition module}

Elevator operation data is very rich, and it is difficult to analyze them all. Therefore, it is necessary to extract elevator fault symptoms from elevator operation data.

\subsubsection{Elevator fault reasoning diagnosis module}

Responsible for completing the function of elevator fault diagnosis using elevator fault symptoms information. This system can integrate a variety of fault diagnosis methods, expert system, SVM method and so on.

\subsubsection{Elevator fault diagnosis results analysis module}

The module analyses and judges the process of elevator fault diagnosis and the result of fault diagnosis in the process.

\subsubsection{Elevator self-learning module}

This module extracts knowledge from accumulated data, and stores knowledge into knowledge base expert system on the premise of ensuring the simplification of knowledge base.

\subsection{Elevator fault reasoning diagnosis module design}

Reasoning diagnosis mechanism is an important fault diagnosis method. The performance of an expert system depends first on the integrity of the knowledge base, that is, whether there is a sufficient and complete rule base; secondly, it is related to the implementation of specific reasoning mechanism. Rule reasoning produces reasoning, which can be either forward reasoning or backward reasoning. The way of reasoning depends on the goal of reasoning and the characteristics of search space. If the goal is to find all inferential conclusions from a set of given facts, positive reasoning should be used; if the goal is to confirm or negate a particular conclusion, reverse reasoning should be used.

\subsection{Elevator self-learning module design}

The maintenance function of knowledge base includes inconsistency and cycle checking of knowledge base. The inconsistency of rules includes redundant rules, contradictory rules, circular rules and dead rules.

\section{Design of elevator fault prediction system}

The parameter data stored continuously in elevator operation database includes important information reflecting the process and development of elevator health. On the basis of a large number of operation data, the change of elevator performance, the evaluation of the remaining service life of the equipment and the detection of potential anomalies provide sufficient time and decision-making basis for the prevention and removal of elevator faults. The elevator fault prediction system is very important.

Elevator fault prediction subsystem module design includes: elevator task management module, elevator data acquisition and pre-processing module, elevator predictor module, result analysis module, result display module, process analysis module.

Elevator task management module is used to customize and manage fault prediction tasks, and supports the coexistence of multiple tasks in the system. Users can customize and activate the required prediction tasks under the guidance of the system. 
The elevator fault data acquisition and pre-processing module mainly reads the corresponding sequence of historical monitoring values from the elevator operation database according to the user-customized task list, and carries out pre-processing operations such as removing outliers, eliminating noise, smoothing filtering and so on.

The elevator predictor module is the core module of the subsystem. It is required to select appropriate methods to construct the prediction model according to the characteristics of the monitoring sequence of equipment performance indicators and to judge the trend of equipment performance. By comparing the predicted value with the limited value of the performance evaluation index, the abnormal performance of the equipment can be found in time, and the abnormal occurrence time can be determined. The module has the function of searching and viewing historical results.

\section{References}

1. Frank P M. Fault diagnosis in dynamic systems using analytical and knowledge-based redundancy-a survey and some new result. Automatics, 1990, 26(3), 459-474.

2. Alvarez J L R, et al. Support Vector method for robust ARMR system identification. IEEE Trans. on Signal Processing, 2004, 52(1):155-164.

3. Rongling Lang. Fault Diagnosis of Airborne Equipments Based on Similarity Search [J]. Chinese Journal of Electronics, 2013, 22(4): 855-860.

4. Suykens J A K, De Brabanter J, Tony Van Gestel. Least Squares Support Vector Machines. World Scientific, 2002. 